\title{
Radiation Models and Radiation Transfer in Hypersonics
}

\author{
M.Y. Perrin ${ }^{*}, 1$, G. Colonna ${ }^{2}$, G. D’Ammando ${ }^{3}$, L.D. Pietanza ${ }^{2}$, Ph. Riviere $^{1}$, A. Soufani ${ }^{1}$ and \\ S. Surzhikov ${ }^{4}$
}

${ }^{l}$ CNRS, UPR 288 Laboratoire d'Energétique Moléculaire et Macroscopique, Combustion (EM2C), Ecole Centrale Paris, Grande Voie des Vignes, 92295 Châtenay-Malabry, France

${ }^{2}$ CNR-IMIP, Via Amendola 122/D, 70126 Bari, Italy

${ }^{3}$ Dipartimento di Chimica, Universitá degli studi di Bari, Via Orabona 4, 70125 Bari, Italy

${ }^{4}$ A. Ishlinsky Institute for Problems in Mechanics, Russian Academy of Sciences, Prospect Vernadskogo 101-1, Moscow 119526, Russia

\begin{abstract}
The paper presents radiation models developed to investigate radiation in entry in Earth, Mars and Jupiter atmospheres. The capacity of ASTEROID computing code to simulate elementary radiative processes, calculate spectral and groups optical properties, and also solve simple radiative heat transfer problems is presented for Earth entry. The large number of radiative processes involved in the radiative flux is put forward. The contributions of the different radiative processes encountered in Mars entry are studied using the HTGR spectroscopic database. The validity of this database with respect to diatomic molecules systems and $\mathrm{CO}_{2}$ infrared radiation is illustrated through experimental validations. The accuracy of statistical narrow-band model to predict radiative flux is illustrated for an afterbody. Finally recent improvements of the model developed for the calculation of radiative properties of high-temperature $\mathrm{H}_{2} / \mathrm{He}$ mixtures representative of Jupiter atmosphere is presented. The model takes into account the most important radiative processes.
\end{abstract}

Keywords: Radiative transfer, re-entry flows, spectroscopic databases.

\section{INTRODUCTION}

During an atmospheric entry, a shock layer is created in front of the vehicle. The hot gases in this shock layer emit a radiation that contributes to the incident flux at the vehicle surface. A correct evaluation of this flux is needed to design the thermal protection shield of the vehicle, especially at very high entry speed. The present paper describes recent advancements and associated numerical codes used for evaluation of radiation in hypersonic flows encountered in Earth, Mars and Jupiter atmospheric entries. Several radiative mechanisms are involved, which give rise to a complex structure of the emission and absorption spectra. Boundbound transitions in atoms and molecules give line spectra. Free-free transitions contribute to a continuous spectrum. Bound-free transitions result in a continuous spectrum with eventually some structures. The selection of the radiative mechanisms and associated spectroscopic data is a critical issue. All the processes which may contribute to emission and absorption have to be considered. Due to the wide temperature range that can be encountered (up to $80,000 \mathrm{~K}$ for entry applications), the spectral range has to cover Infrared (IR) to Vacuum Ultra-Violet (VUV). Ideally, the

*Address correspondence to this author at the CNRS, UPR 288 Laboratoire d'Energétique Moléculaire et Macroscopique, Combustion (EM2C), Ecole Centrale Paris, Grande Voie des Vignes, 92295 Châtenay-Malabry, France;

Tel: 331411310 62; Fax: 331470280 35;

E-mail: marie-yvonne.perrin@ecp.fr monochromatic local emission and absorptions coefficients, which depend on the the internal sates of the gas in the nonequilibrium shock layer, have to be known to determine the radiative flux. However, models of radiative properties may be of interest for practical applications. It should be noted that radiation may also modify the gas dynamics. The emitted photons can either leave the flow or can be reabsorbed, contributing to the transport of energy. Under some conditions, the processes of emission and absorption of photons have to be included in the equations describing the evolution of atomic and molecular internal states. Modeling radiation in hypersonics is not an easy task, which explains the development of several computer codes and spectral databases in the last decades: NEQAIR [1], LORAN [2], SPRADIAN [3], MONSTER [4], SPECAIR [5], PARADE [6], HARA [7], GPRD [8].

The paper presents recent efforts of three groups to progress in the field. Section 2 gives a brief description of the ASTEROID computing code developed in Russia for the numerical simulation of elementary radiative processes, calculation of spectral and groups optical properties, and also for solving simple radiative heat transfer problems. As an example, the calculated half-spherical emissivity of high temperature air, which can be used to estimate radiative heating of space vehicles entering into atmospheric dense layers, is presented. Section 3 presents the HTGR spectroscopic database developed in France which aims at modelling high resolution radiation. Applications to $\mathrm{CO}_{2} / \mathrm{N}_{2}$ 
mixtures representative of Mars atmosphere are presented. Section 4 presents recent improvements of the model developed in Italy for the calculation of radiative properties of high-temperature $\mathrm{H}_{2} / \mathrm{He}$ mixtures representative of Jupiter atmosphere, taking into account the most important radiative processes.

\section{ASTEROID AND EARTH ATMOSPHERE ENTRY PLASMA}

Notwithstanding the fact that the theory and the practice for the calculation of elementary and statistical radiative properties of low temperature plasma and hot gases have been developing for more than a half century [9-13], the development of reliable spectral optical models for radiative gas dynamics and heat transfer application is still an issue. Modern computing technologies give new methods for improvement of calculations [14, 15]. The ASTEROID computing code has been developed for solving a wide class of problems that are linked with radiation heat transfer in hot gases and low-temperature plasmas namely:

1. numerical simulation of elementary radiation processes cross-sections;

2. creation of spectral, multi-group, integral and combined models of absorption and emission coefficients;

3. creation of the optimum multi-group radiative models for low-temperature plasma and hot gases;

4. numerical simulation of spectral radiative heat transfer in simple cases i.e of plane, cylindrical and spherical volumes.

The following sections give a brief description of this code and a few examples of application.

\subsection{Brief Description of Models and Code}

The optical and radiative models can be created for temperatures $T$ ranging from $300 \mathrm{~K}$ to $20,000 \mathrm{~K}$ and more, for pressures $p$ up to $100 \mathrm{~atm}$ and for wave numbers in the range $1000-500,000 \mathrm{~cm}^{-1}$. Presently, the ASTEROID code contains spectroscopic information and numerical simulation models for the following chemical elements: $\mathrm{H}, \mathrm{He}, \mathrm{C}, \mathrm{N}, \mathrm{O}$, $\mathrm{Si}, \mathrm{Ar}, \mathrm{Na}, \mathrm{Mg}, \mathrm{Al}, \mathrm{K}, \mathrm{Ca}$. The ASTEROID code is available in two versions.

The first one is actually the computing platform intended for comparative investigation of different quantum mechanical and quasi-classical models of elementary radiative processes. It is well known that a specific property of radiative gas dynamics is the necessity to take into account a large number of elementary radiative processes in a wide temperature region. To describe each of individual radiative processes a set of theoretical and computational models have been developed, from detailed ab-initio models down to approximate quasi-classical and half-empirical ones. Each of the models has a defined field of application. Most universal ab-initio quantum mechanical Hartree-Fock models of multi-channel interactions are intended for description of the fine structure of absorption and emission spectra. These models are very time-consuming, and therefore could not be included into complex radiative gas dynamic codes. On the other side, simplified semi-empirical and quasi-classical models can be used together with gas dynamic and kinetic codes, because of their high computational efficiency, but, unfortunately, they could not describe several significant peculiarities of radiative processes. Consequently, the creation of integrated radiative gas dynamic codes, which include models of gas dynamics, chemical and physical kinetics, as well as radiation heat transfer models and models of spectral (or group) optical properties, always conjugates with choice of alternative approaches.

The second version of ASTEROID, which is called ABSORB, is a significantly simplified version of the first one. ABSORB is intended for calculation of spectral and group optical models in the frame of radiative gas dynamic codes. It is assumed that models included into the code have been tested by comparison with other alternative models of ASTEROID code, as well as with available experimental data. Initial data for ABSORB (temperature, species concentrations, total pressure) are prepared after solution of gas dynamic equations, species and energy conservation equations. As for initial conditions for ASTEROID code, three files for the initial data are accordingly available. The first one contains initial data, specifying chemical composition of the considered mixture of gases and a scale of temperatures; the second file contains initial data, specifying number and the sizes of each spectral group, and also number of points on wave number in limits of each spectral group; and the third file contains initial data, specifying geometry of volume, and also distribution of temperature in this volume.

The principles of fundamental spectroscopy form the basis of the ASTEROID code. Therefore a database of energy levels of atoms and ions [16, 17], as well as a database of energy levels of diatomic molecules and ions [18], also used for the calculation of probabilities of elementary radiative processes, and the atomic and molecular lines parameters are included.

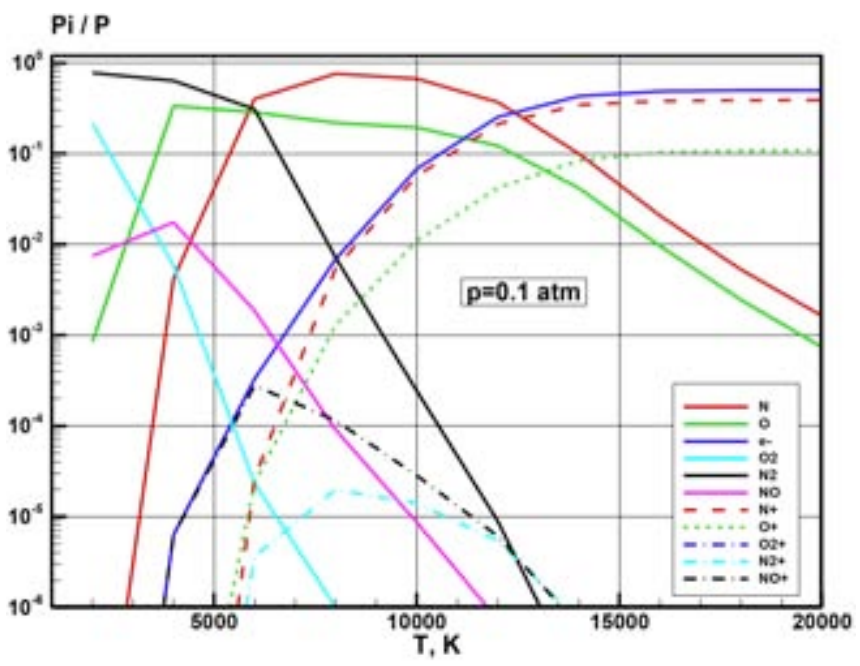

Fig. (1). Molar fractions of air species $\left(x_{i}=\frac{p_{i}}{p}\right)$ at pressure $p=0.1 \mathrm{~atm}$.

The ASTEROID code creates data files containing the information for the equilibrium chemical composition, optical group models, and also group and integrated characteristics of radiation transfer. Fig. (1) shows an example of calculations of equilibrium chemical composition 
of high temperature air at pressure $p=0.1$ atm (double ionization of atoms is not taken into account).

The ASTEROID computing platform contains several auxiliary programs, which cannot be integrated in the computing system because they are highly laborious or cumbersome to use. The results obtained by these programs are used to create local databases, or to compare with results obtained by any alternate programs. These programs are dedicated to the calculation of:

\section{Franck-Condon-factors;}

2. parameters of RKR-potential of diatomic molecules;

3. rotational lines parameters of diatomic molecules;

4. vibrational wave functions of diatomic molecules;

5. radial wave functions of atoms and ions;

6. parameters for atoms and ions lines;

7. fine structure for the absorption and emission spectrum of diatomic molecules;

8. absorption coefficients for diatomic molecules averaged on rotational and vibrational structures;

9. absorption coefficients averaged on the rotational structure of multi-atomic molecules in the infrared area of a spectrum;

10. absorptivity (or transmissivity) of non-uniform gas layers assuming thin rotational structure of a spectrum of diatomic molecules. These codes use "line-by-line" methods, and also various random models;

11. absorptivity (or transmissivity) of non-uniform layers of low-temperature plasma based on atomic lines. These codes use "line-by-line" methods, and also various random models.

The ASTEROID computing platform allows to investigate not only basic models of elementary radiative processes but also to conjugate its simplified version (code ABSORB) with other models of radiative gas dynamics.

\subsection{Use of Integral Half-Spherical Emissivities to Estimate Radiative Heating}

When solving applied problems of aerophysics, there is often a need of fast estimations of radiative heating of entering space vehicles without solving the full system of radiative gas dynamic equations. This can be done for 'standard' mixtures like air, $\mathrm{CO}_{2}-\mathrm{N}_{2}$ (97\%-3\%), etc. The method includes a few simple steps. On a first step, an estimation of shock wave stand-off distance $\delta$ is calculated with the approximate formula [19]:

$$
\delta=\frac{\varepsilon R}{1+\sqrt{8 \varepsilon / 3}}, \varepsilon=\frac{\rho_{\infty}}{\rho_{w}},
$$

where $\rho_{\infty}$ and $\rho_{w}$ are the densities in the free stream flow and at the surface. $R$ is the blunt body nose radius.

On a second step, temperature and pressure are calculated in the equilibrium zone of the shock layer behind the shock wave. A model of thermodynamic equilibrium can be used for this purpose. Assuming an homogeneous volume, the half-spherical integral emissivity $\varepsilon_{0}$ of the corresponding 'standard' mixture is calculated from the shock layer depth, approximated by $\delta$, its temperature and pressure according to:

$\varepsilon_{0}=\frac{\pi}{\tilde{\sigma} T^{4}} \int_{\sigma_{\min }}^{\sigma_{\max }}\left(1-\exp \left(-\kappa_{\sigma} \delta\right)\right) I_{\sigma}^{b} d \omega$,

where $\kappa_{\sigma}$ is the spectral absorption coefficient and $\sigma_{\min }$ and $\sigma_{\max }$ are the wave numbers corresponding to the boundaries of the heat radiation spectral region. $I_{\sigma}^{b}$ is the equilibrium intensity given by the Planck's law and $\tilde{\sigma}$ is the StephanBoltzmann constant.

The radiative flux can be determined by:

$q_{\text {rad }}=\varepsilon_{0} \tilde{\sigma} T^{4}$

Spectral absorption coefficients $\kappa_{\sigma}$ of high temperature air, calculated with the code ASTEROID in $10^{6}$ spectral points, are presented in Figs. $(\mathbf{2}, \mathbf{3})$ for two temperatures. These thermodynamic points are quite typical for problems of radiative heating of entering space vehicles. Halfspherical emissivities of high temperature air are presented in Figs. (4-6). Each of the figures shows half-spherical emissivity at three pressures calculated with and without atomic lines structure. It should be noted that taking into account the line structure of emission and absorption spectra is one of unsolved problems of radiative gas dynamics with strong radiative-gas dynamic interaction.

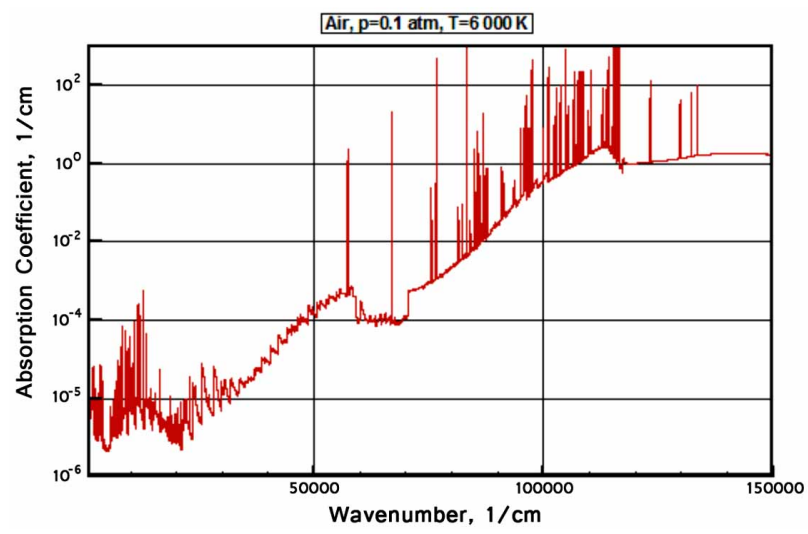

Fig. (2). Spectral absorption coefficient of air at $p=0.1 \mathrm{~atm}$ and $T=6,000 \mathrm{~K}$.

Figs. (7-9) show cumulative distributions defined by

$$
Q(\sigma)=\frac{\pi}{\tilde{\sigma} T^{4}} \int_{\sigma_{\min }}^{\sigma}\left(1-\exp \left(-\kappa_{\sigma} \delta\right)\right) I_{\sigma}^{b} d \omega
$$

where $\sigma_{\text {min }}=1000 \mathrm{~cm}^{-1}$ is the minimal wave number for integration. These cumulative functions allow to demonstrate the most significant spectral subregions contributing to the integral emissivity. For example, at temperature $T=6,000 \mathrm{~K}$ and pressure $p=0.1$ atm (see cumulative function in Fig. 7) radiative processes in the spectral subregion $10,000-55,000 \mathrm{~cm}^{-1}$ 


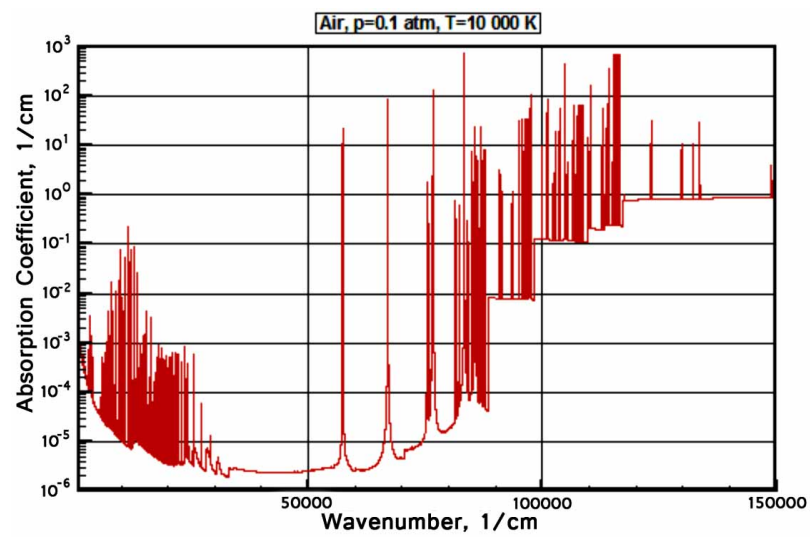

Fig. (3). Spectral absorption coefficient of air at $p=0.1 \mathrm{~atm}$ and $T=10,000 \mathrm{~K}$.

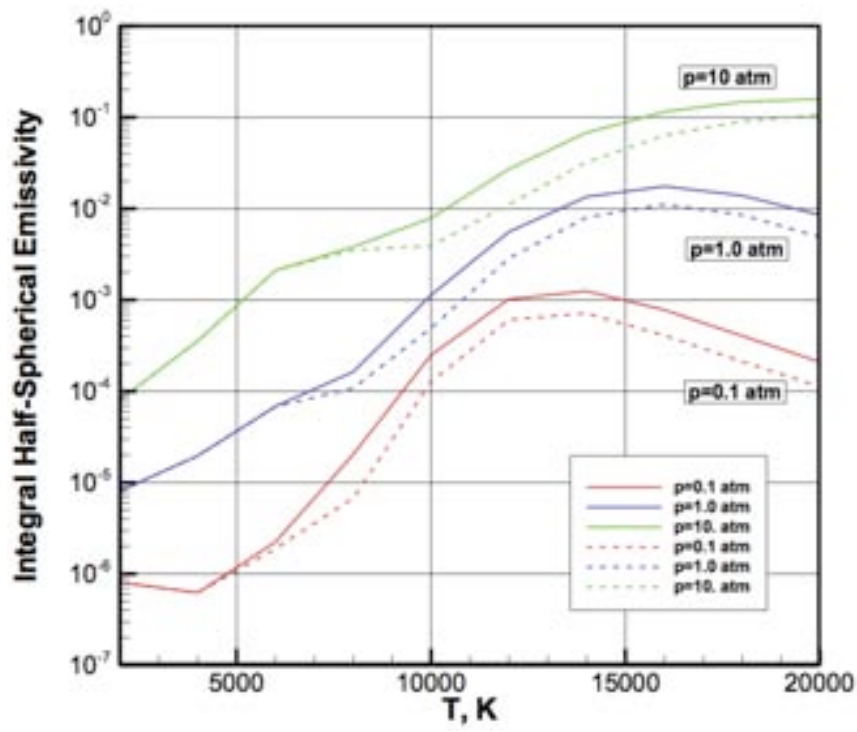

Fig. (4). Half-spherical integral emissivity of high temperature air with (solid lines) and without (dashed lines) atomic lines at $R=1 \mathrm{~cm}$.

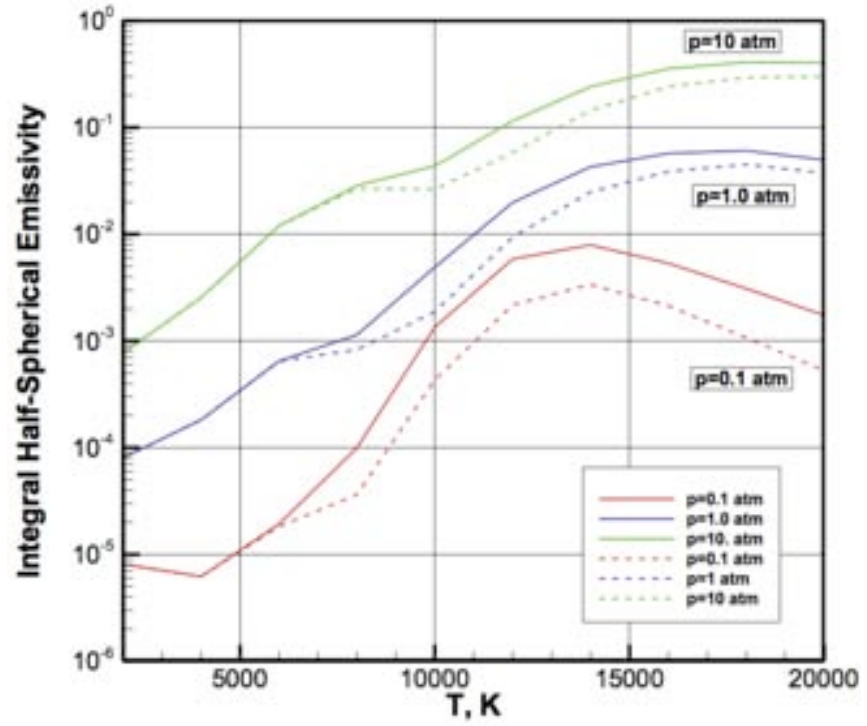

Fig. (5). Half-spherical integral emissivity of high temperature air with (solid lines) and without (dashed lines) atomic lines at $R=10 \mathrm{~cm}$.

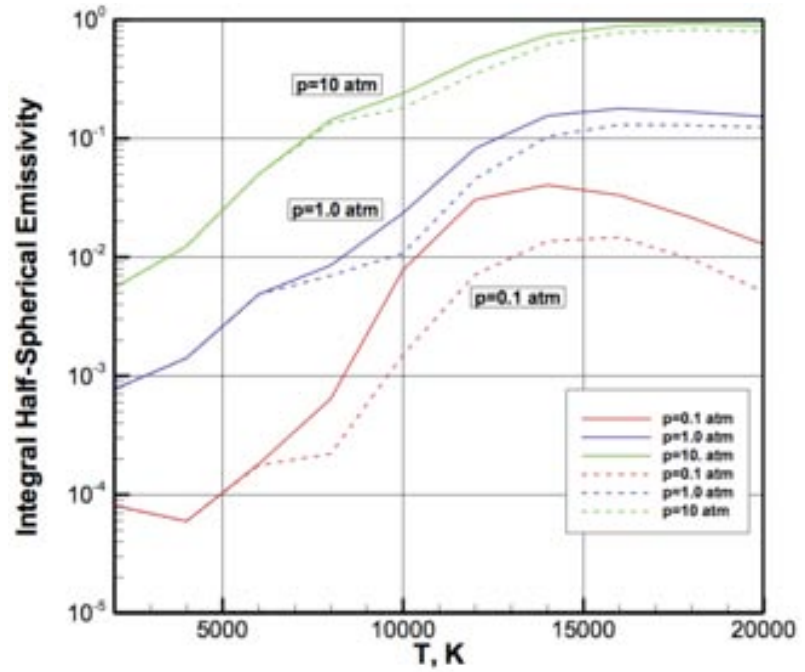

Fig. (6). Half-spherical integral emissivity of high temperature air with (solid lines) and without (dashed lines) atomic lines at $R=100 \mathrm{~cm}$.

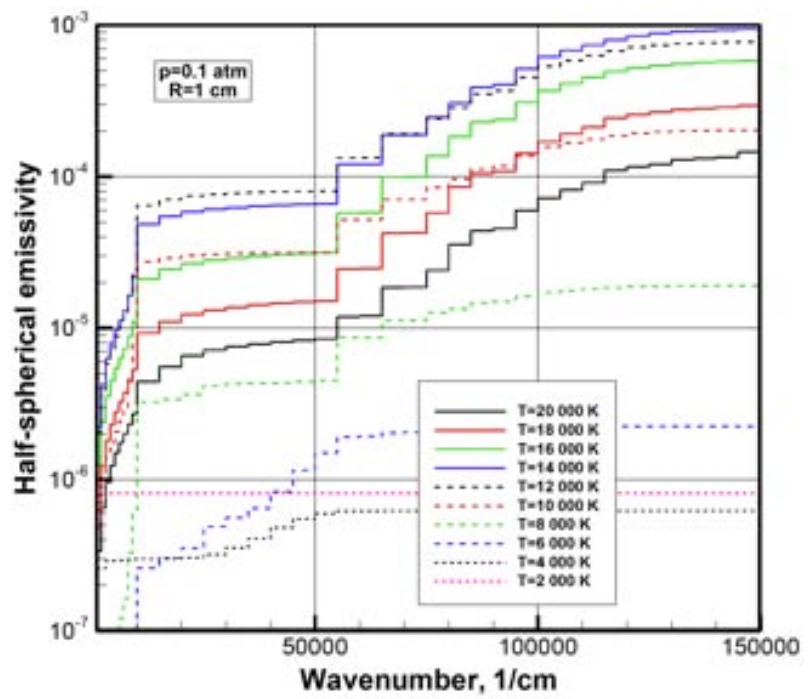

Fig. (7). Cumulative functions of spectral half-spherical emissivity at $p=0.1 \mathrm{~atm}$ and $R=1 \mathrm{~cm}$.

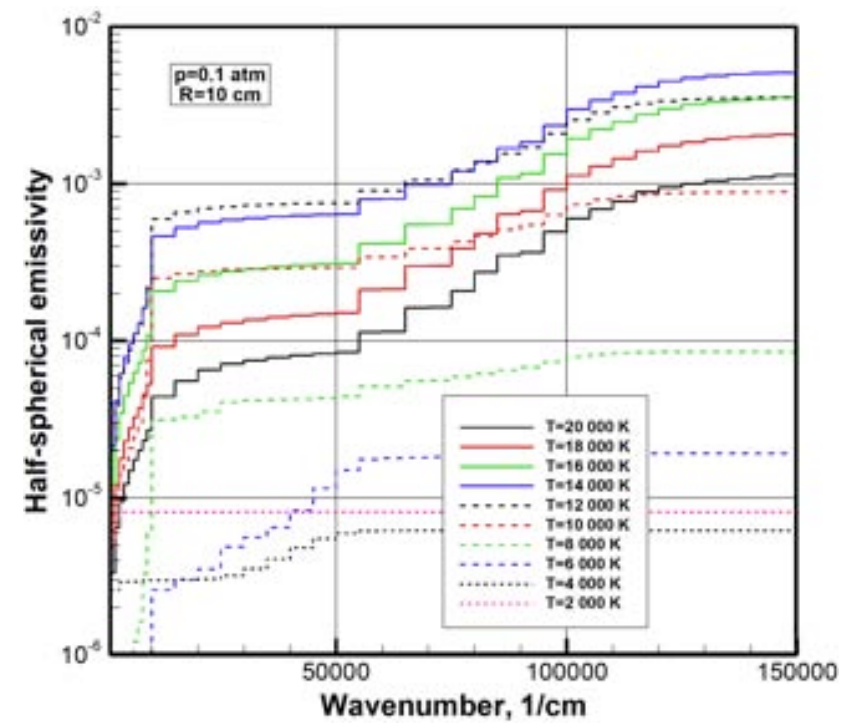

Fig. (8). Cumulative functions of spectral half-spherical emissivity at $p=0.1 \mathrm{~atm}$ and $R=10 \mathrm{~cm}$. 


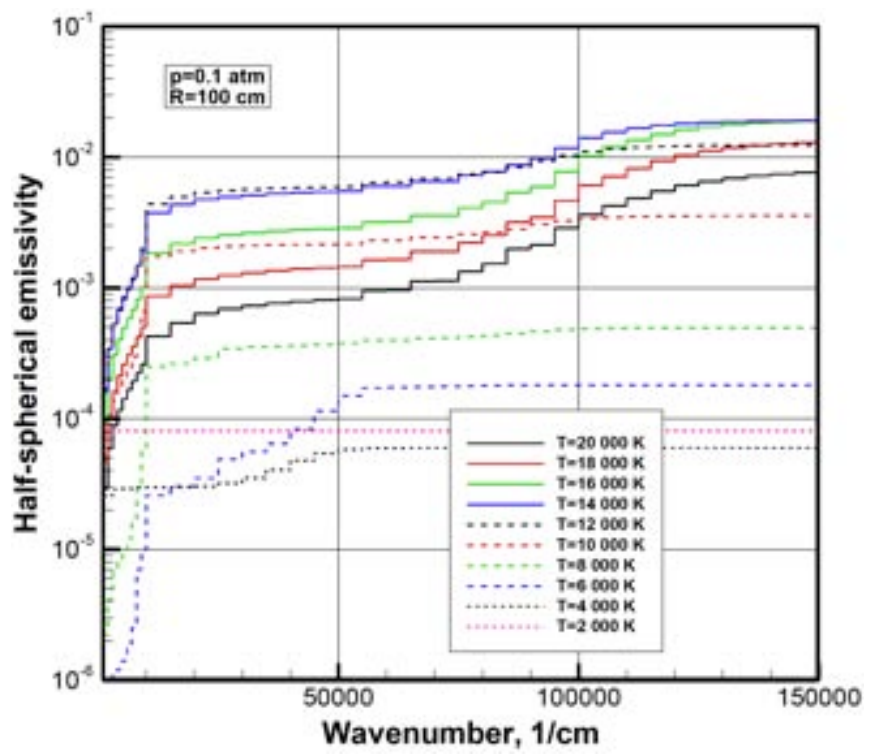

Fig. (9). Cumulative functions of spectral half-spherical emissivity at $p=0.1 \mathrm{~atm}$ and $R=100 \mathrm{~cm}$.

give general contribution to integral emissivity, while at $T=12,000 \mathrm{~K}$ radiative processes located in spectral subregion $60,000-120,000 \mathrm{~cm}^{-1}$ become most significant. The spectral distributions of individual radiation processes, shown in Figs. $(\mathbf{1 0}, \mathbf{1 1})$, allow to identify the most significant radiative

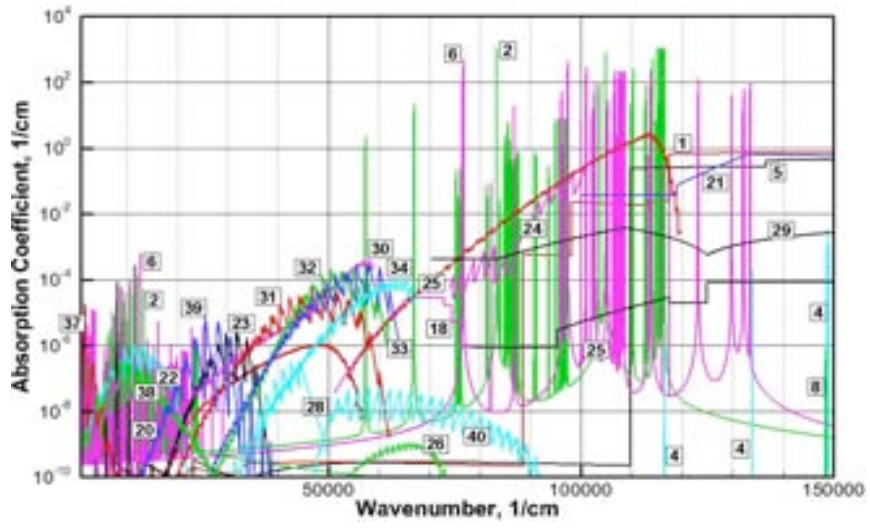

Fig. (10). Partial contribution of individual radiative processes to the total absorption coefficient of high temperature air at $p=0.1 \mathrm{~atm}$, $T=6,000 \mathrm{~K}$.

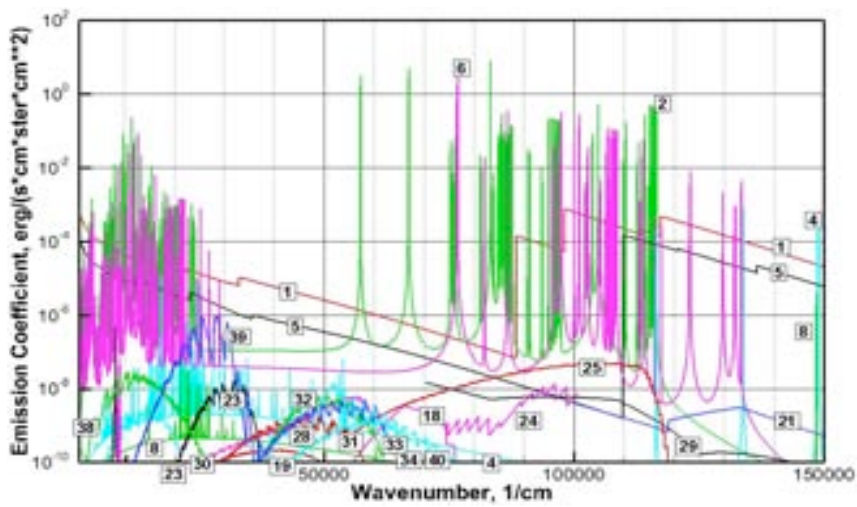

Fig. (11). Partial contribution of individual radiative processes to the total emission coefficient of high temperature air at $p=0.1 \mathrm{~atm}$, $T=12,000 \mathrm{~K}$. processes Table $\mathbf{1}$ details the elementary processes represented in Figs. $(\mathbf{1 0}, \mathbf{1 1})$. In the case under consideration photoionization from bound states of atoms $\mathrm{N}$ and $\mathrm{O}$, as well as resonant atomic lines are most significant at $T=12,000 \mathrm{~K}$. At $T=6,000 \mathrm{~K}$, the electronic bands of diatomic molecules and radicals $\left(\mathrm{N}_{2}, \mathrm{O}_{2}, \mathrm{NO}\right)$ are most significant.

Table 1. Elementary processes in Figs. $(10,12)$.

\begin{tabular}{|c|c|c|c|}
\hline Number & Elementary Process & Number & Elementary Process \\
\hline \hline 1 & $\mathrm{~N}:$ bound - free & 26 & $\mathrm{~N}_{2}: A^{3} \Sigma_{\mathrm{u}}^{+}-X^{1} \Sigma_{\mathrm{g}}^{+}$ \\
\hline 2 & $\mathrm{~N}:$ atomic lines & 28 & $\mathrm{~N}_{2}: a^{1} \Pi_{\mathrm{g}}-X^{1} \Sigma_{\mathrm{g}}^{+}$ \\
\hline 4 & $\mathrm{~N}^{+}:$atomic lines & 29 & NO: photoionization \\
\hline 5 & $0:$ bound - free & 30 & NO: $B^{2} \Pi-X^{2} \Pi$ \\
\hline 6 & $0:$ atomic lines & 31 & $\mathrm{NO}: A^{2} \Sigma^{+}-X^{2} \Pi$ \\
\hline 8 & $0^{+}:$atomic lines & 32 & $\mathrm{NO}: C^{2} \Pi-X^{2} \Pi$ \\
\hline 18 & $\mathrm{O}_{2}:$ photodissociation & 33 & $\mathrm{NO}: D^{2} \Sigma^{+}-X^{2} \Pi$ \\
\hline 19 & $\mathrm{O}_{2}: B^{3} \Sigma_{\mathrm{u}}^{-}-X^{3} \Sigma_{\mathrm{g}}^{-}$ & 34 & $\mathrm{NO}: B^{\prime 2} \Delta-X^{2} \Pi$ \\
\hline 21 & $\mathrm{~N}_{2}:$ photoionization & 37 & $\mathrm{NO}^{2} X^{2} \Pi-X^{2} \Pi$ \\
\hline 22 & $\mathrm{~N}_{2}: B^{3} \Pi_{\mathrm{g}}-A^{3} \Sigma_{\mathrm{u}}^{+}$ & 38 & $\mathrm{~N}_{2}^{+}: \mathrm{A}^{2} \Pi_{\mathrm{u}}-\mathrm{X}^{2} \Sigma_{\mathrm{g}}^{+}$ \\
\hline 23 & $\mathrm{~N}_{2}: C^{3} \Pi_{\mathrm{u}}-B^{3} \Pi_{\mathrm{g}}$ & 39 & $\mathrm{~N}_{2}^{+}: B^{2} \Sigma_{\mathrm{u}}^{+}-X^{2} \Sigma_{\mathrm{g}}^{+}$ \\
\hline 24 & $\mathrm{~N}_{2}: b^{1} \Pi_{\mathrm{u}}-X^{1} \Sigma_{\mathrm{g}}^{+}$ & 40 & $\mathrm{~N}_{2}^{+}: C^{2} \Sigma_{\mathrm{u}}^{+}-X^{2} \Sigma_{\mathrm{g}}^{+}$ \\
\hline 25 & $\mathrm{~N}_{2}: b^{\prime 1} \Sigma_{\mathrm{u}}^{+}-X^{1} \Sigma_{\mathrm{g}}^{+}$ & & \\
\hline
\end{tabular}

\section{HTGR AND MARS ATMOSPHERE ENTRY PLASMA}

\subsection{HTGR Radiation Database}

The HTGR spectroscopic database has been originally developed to describe radiative processes in air $[20,21]$. Later, it has been extended to include radiative processes observed in $\mathrm{CO}_{2}-\mathrm{N}_{2}$ plasma [22] and is continuously complemented with new species and radiative processes. Atomic species line-strengths and line-positions are taken from the NIST database [17]. As many atomic lines can be optically thick in the considered applications, their line shapes have to be carefully determined. Systematic calculations have been performed in the impact approximation to account for the different collisional broadening mechanisms [23]. The database includes 35 electronic systems of $\mathrm{N}_{2}, \mathrm{O}_{2}, \mathrm{NO}, \mathrm{N}_{2}^{+}, \mathrm{CO}, \mathrm{CO}^{+}, \mathrm{C}^{2}$ and $\mathrm{CN}$ molecules. A systematic approach is used to calculate diatomic spectroscopic data [20]. Line positions are taken from the literature in order to have a good accuracy. The RKR procedure is used to reconstruct the intramolecular potential. The radial Schrödinger equation is solved to get rotationless-vibrational wave functions which are combined to critically selected ab-initio or experimental electronic transition moment functions to obtain the line strengths. The atomic species photoionization cross sections are taken from the Opacity Project (OP) [24]. Molecular photoionization, molecular photodissociation and negative atomic ions photodetachment are modelled pragmatically [21, 22]. Freefree transitions are included. The knowledge of very high 
temperature absorption and emission spectra of triatomic molecules like $\mathrm{CO}_{2}$ remains a challenge. The recent CDSD4000 database [25] has been selected for modeling $\mathrm{CO}_{2}$ IR emission.

The HTGR database allows to calculate the spectral characteristics, in particular the spectral emission and absorption coefficients, $\eta_{\sigma}$ and $\kappa_{\sigma}$, on a wide spectral range, $1,000-200,000 \mathrm{~cm}^{-1}$, by a line-by-line approach from local thermochemical state and level populations. Fig. (12) shows the absorption spectrum of equilibrium $\mathrm{CO}_{2}(97 \%)-\mathrm{N}_{2}$ (3\%) plasma, which composition is representative of Mars atmosphere, at 1 atm and $5000 \mathrm{~K}$. Fig. (13) shows the optically thin emission defined by:

$S_{R}=\int \kappa_{\sigma} I_{\sigma}^{b} d \sigma$

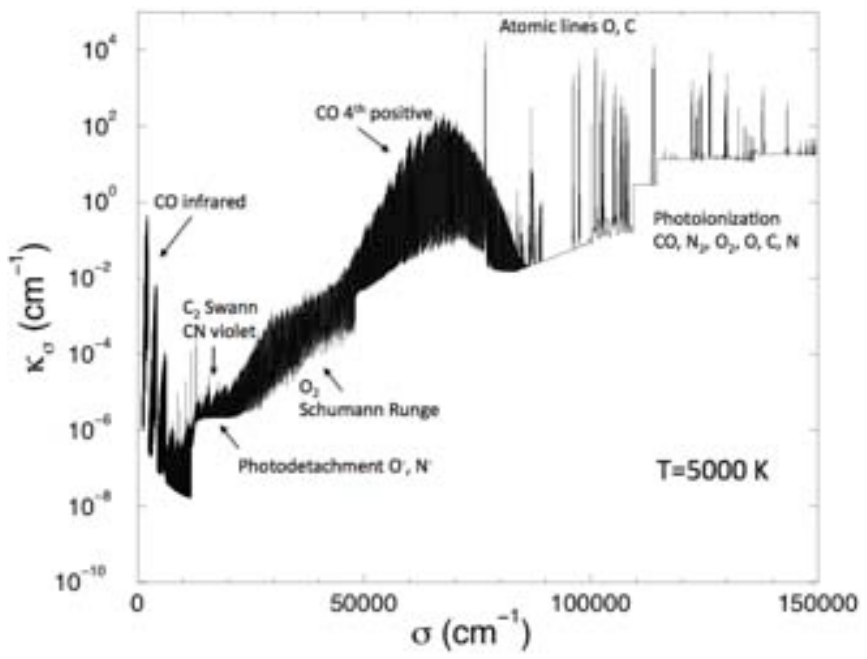

Fig. (12). Absorption spectrum of equilibrium $\mathrm{CO}_{2}(97 \%)-\mathrm{N}_{2}(3 \%)$ plasma at $1 \mathrm{~atm}$ and $5,000 \mathrm{~K}$.

Are also shown in Fig. (13) the contributions due to atomic bound-bound processes, diatomic bound-bound processes, continuum processes and $\mathrm{CO}_{2}$ infrared radiation. In the backside of a capsule entering Mars atmosphere, the temperature is limited to $4,000 \mathrm{~K}$, the radiation will be due mainly to $\mathrm{CO}_{2}$. In the front side, the temperature in the shock layer is typically in the range $[2,000-7,000] \mathrm{K}$. The contribution of $\mathrm{CO}_{2}$ infrared radiation is important up to $4000 \mathrm{~K}$; the radiation is due to diatomic bound-bound processes above. These two types of radiative processes are more thoroughly discussed in the following sections.

\subsection{Carbonaceous Diatomic Molecules}

The spectra of carbonaceous diatomic molecular sytems have been studied both theoretically and experimentally. The most important electronic systems for Martian entries are those of $\mathrm{CO}$ (Infrared, fourth posistive $A^{1} \Pi-X^{1} \Sigma^{+}$, Hopfield-Birge $B^{1} \Sigma^{+}-X^{1} \Sigma^{+}$, and third Positive $\left.b^{3} \Sigma^{+}-a^{3} \Pi\right)$, of $\mathrm{CN}$ (Red $A^{2} \Pi_{i}-X^{2} \Sigma^{+}$, Violet $B^{2} \Sigma^{+}-X^{2} \Sigma^{+}$, and LeBlanc $\left.B^{2} \Sigma^{+}-A^{2} \Pi_{i}\right)$, of $\mathrm{CO}^{+}$(Comet-tail $A^{2} \Pi_{i}-X^{2} \Sigma^{+}$, Baldet-Johnson $B^{2} \Sigma^{+}-A^{2} \Pi_{i}$, and First Negative
$B^{2} \Sigma^{+}-X^{2} \Sigma^{+}$), and of $\mathrm{C}_{2}$ (Philips $A^{1} \Pi_{u}-X^{1} \Sigma_{g}^{+}$, Mulliken $D^{1} \Sigma_{u}^{+}-X^{1} \Sigma_{g}^{+}$, Deslandres-D'Azambuja $C^{1} \Pi_{g}-A^{1} \Pi_{u}$, Swan $d^{3} \Pi_{g}-a^{3} \Pi_{u}, \quad$ Ballik-Ramsay $b^{3} \Sigma_{g}^{-}-a^{3} \Pi_{u}, \quad$ and FoxHerzberg $\left.e^{3} \Pi_{g}-a^{3} \Pi_{u}\right)$. Theoretical calculations have been carried out using the RKR method for internuclear potential reconstruction, a spectral Chebyshev method for the determinantion of vibrational wave functions from the radial Schrödinger equation, and using up-to-date available electronic transition moment functions [22].

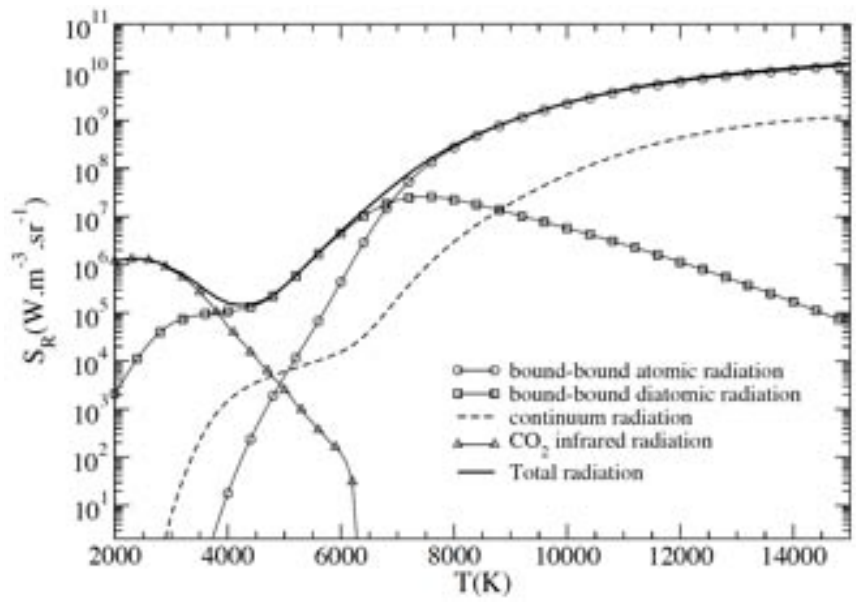

Fig. (13). Optically thin emission of bound-bound atomic, boundbound diatomic, continuum and $\mathrm{CO}_{2}$ infrared mechanisms for a $\mathrm{CO}_{2}(97 \%)-\mathrm{N}_{2}(3 \%)$ plasma at $1 \mathrm{~atm}$.

Some of these systems have been studied experimentally using optical emission spectroscopy and a microwave plasma flow confined in a quartz tube [26]. Fig. (14) shows an example of comparison between absolute local (Abel inverted) measured emission coefficient and the predicted coefficient from HTGR databse for the $\mathrm{C}_{2}$ Swan system. The spectral range in this figure corresponds to vibrational bands characterized by $\Delta v=v^{\prime}-v^{\prime \prime}=1$. The agreement between

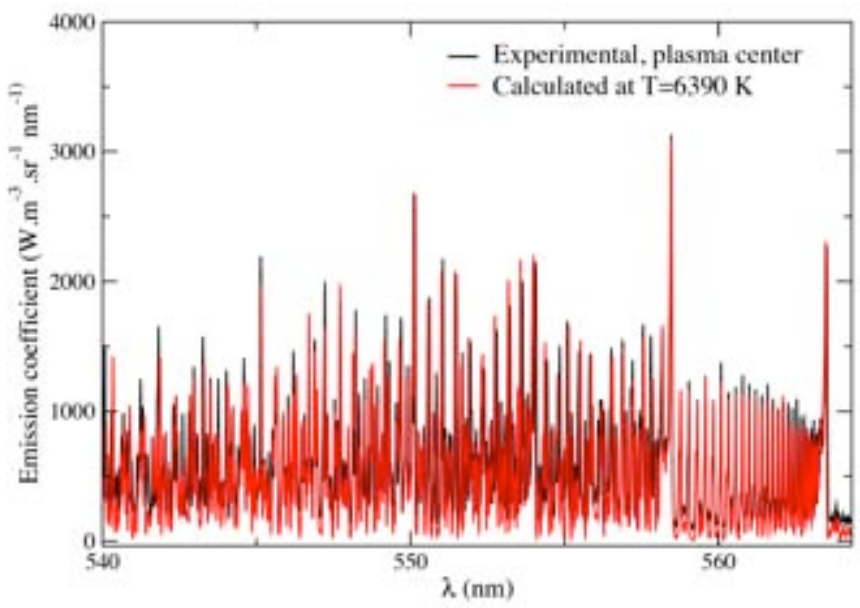

Fig. (14). Comparison between the absolute levels of emission coefficient at the center of a $\mathrm{CO}_{2}$ plasma and $6 \mathrm{~mm}$ above the exit of the microwave cavity, and the calculated one, for the $C_{2}$ Swan system, $\Delta v=1$ region. The experimental setup is described in [26]. 
the predictions and the experimental calibrated spectrum is very satisfactory. The HTGR database possesses a spectroscopic accuracy which allows its use in optical diagnostics. Fig. (15) shows an example of comparisons between normalized spectra in the spectral range corresponding to the $\mathrm{CN}$ violet $\Delta v=-1$ vibrational bands. The adjustment of the measured spectrum enables quite accurate determination of vibrational and rotational temperatures.

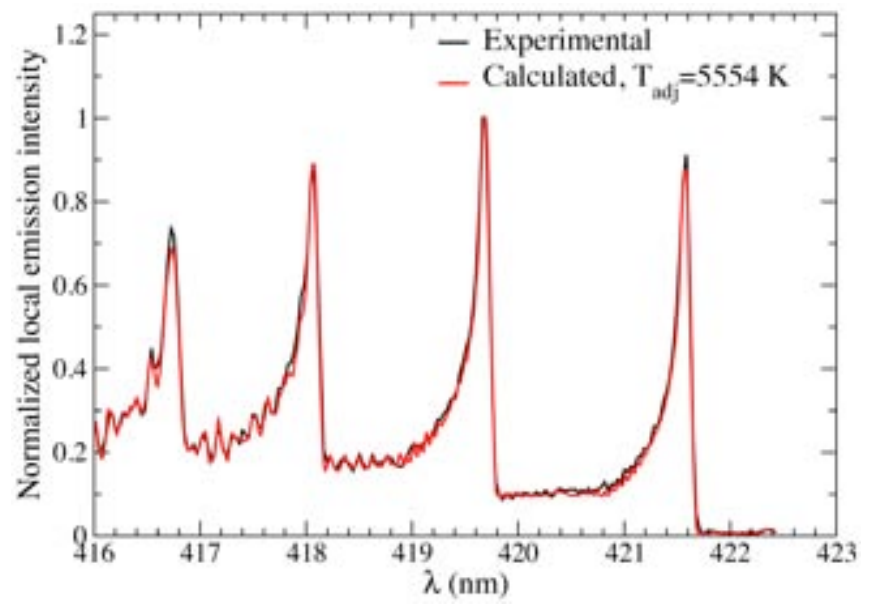

Fig. (15). Normalized experimental and adjusted local emission coefficient in a $\mathrm{CO}_{2}-\mathrm{N}_{2}$ plasma. The peaks correspond to vibrational bands $\Delta v=-1$ of the $\mathrm{CN}$ violet system.

\section{3. $\mathrm{CO}_{2}$ Infrared Radiation}

As shown above in Fig. (13), $\mathrm{CO}_{2}$ IR radiation remains very important at temperatures as high as $4000 \mathrm{~K}$ although its concentration is quite small. Its contribution is then expected to be important for high velocity entries in planetary atmospheres containing $\mathrm{CO}_{2}$. We have studied the accuracy of various available $\mathrm{CO}_{2}$ databanks by comparing their predictions to measurements $[27,28]$. To this end, an experimental setup combining a microwave plasma torch and a high resolution Fourier transform spectrometer has been designed. $\mathrm{CO}_{2}$ and $\mathrm{CO}_{2}-\mathrm{N}_{2}$ flows were excited by a microwave discharge inside quartz or sapphire tubes and temperature and concentration distributions were inferred from $\mathrm{CO}$ overtone emission in the spectral range [4000-4360 $\left.\mathrm{cm}^{-1}\right]$. The line of sight integrated emission spectra were then compared to the predictions from CDSD4000 [25] and HITELOR [29] spectroscopic databases in the 4.3 and $2.7 \mu \mathrm{m}$ regions. A comparison between measurements and predictions is shown in Fig. (16) for a pure $\mathrm{CO}_{2}$ plasma confined in a saphire tube and $20 \mathrm{~mm}$ above the exit of the microwave discharge. It appears that the recent CDSD-4000 database is more accurate than HITELOR, especially in the $2.7 \mu \mathrm{m}$ region. The high experimental uncertainties in the range $\left[2,200-2,400 \mathrm{~cm}^{-1}\right]$ are due to important uncertainties on the temperature distribution in the cold peripheral regions of the flow.

The CDSD-4000 gathers 573,881,316 rovibrational transitions, when considering only the main isotopologue ${ }^{12} \mathrm{C}^{16} \mathrm{O}_{2}$. A correct description of each spectral line profile associated to these transitions requires about $10^{6}$ spectral points to predict accurately the radiative transfer in the IR spectral range, at atmospheric pressure. At lower pressures such as encountered in Martian entry problems, spectral line profiles are dominated by Doppler broadening, and become therefore much narrower, requiring about 10 times more spectral points. Such spectral high resolution predictions of radiative transfer will therefore require a large amount of $\mathrm{CPU}$ time, in particular when complex 3D geometries are considered.

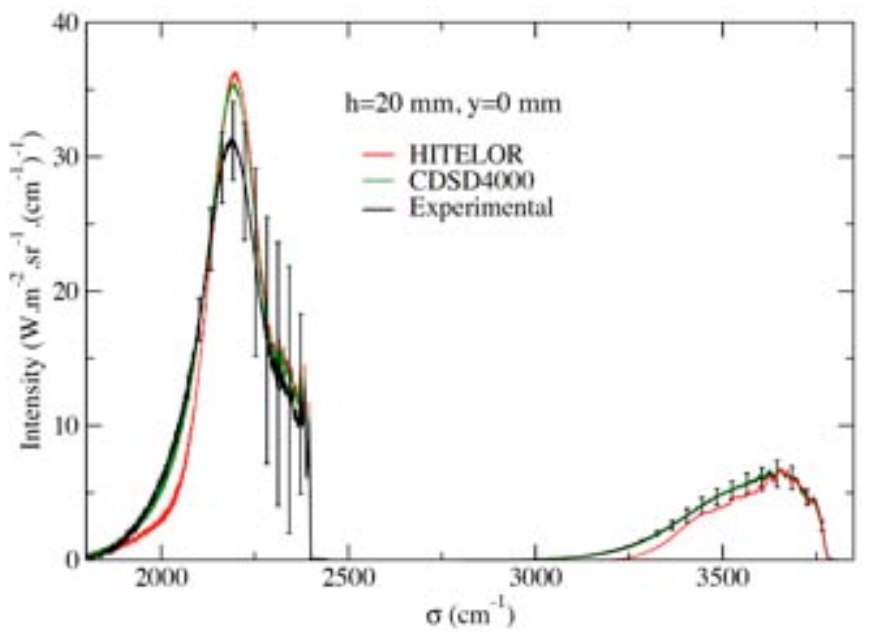

Fig. (16). Comparison between experimental and calculated line-ofsight integrated intensity emitted by a $\mathrm{CO}_{2}$ plasma in the infrared. For legibility, the high resolution spectra are convolved with a rectangular function of width $10 \mathrm{~cm}^{-1}$. Adapted from [28].

Statistical Narrow Band (SNB) models [30] enable to easily calculate the mean transmissivities of an optical path, averaged over spectral narrow-bands, whose widths are a few $10 \mathrm{~cm}^{-1}$, from only a few parameters: the mean absorption coefficient inside each narrow-band, and a parameter characterizing the overlapping of absorption lines within the narrow-band. Such models enable to drastically reduce the required number of "spectral" resolutions of the radiative transfer equation --- about 300 narrow-bands of width $25 \mathrm{~cm}^{-1}$ are sufficient to cover the IR spectral range where $\mathrm{CO}_{2}$ is active. We have recently obtained updated SNB parameters for $\mathrm{CO}_{2}$, based on the CDSD-4000 database at atmospheric pressure [31], and also in pure Doppler broadening regime [32]. These parameters have been calculated from least square adjustments between CDSD4000 based line-by-line and SNB predictions of mean transmissivities of columns for various optical thicknesses, and tabulated in a wide range of temperature between 300 and $5000 \mathrm{~K}$.

As an example of application, these SNB parameters have been used to calculate the incident radiative flux at some locations of the afterbody of the Viking capsule during its entry in Mars atmosphere [33]. 3D aerothermal fields used to perform these radiative transfer calculations were provided from [34]. No coupling with radiative transfer was considered. Radiative transfer calculations were carried out, assuming local thermal equilibrium at the rotation-translation temperature, and only considering Doppler broadening of spectral lines. Fig. (17) shows the spectral distribution in the $4.3 \mu \mathrm{m}$ region of the radiative flux at the thermocouple location on the outer aluminum cone of the Viking afterbody (see details in [33]). Three flight times are considered. SNB model results compare successfully with the reference results 
obtained from a spectral high resolution approach of radiative transfer and line-by-line calculations (LBL). Fig. (17) shows also results obtained from the SNB model, under the weak limit approximation (SNB weak), which consists in neglecting the spectral correlation of gaseous radiative properties within each narrow-band, and thus in just using a mean absorption coefficient (box model); such simplification introduces large discrepancies in this case, confirming the need of using a full correlated model like SNB. More details on these calculations and discussions on the retained approximations may be found in [33].

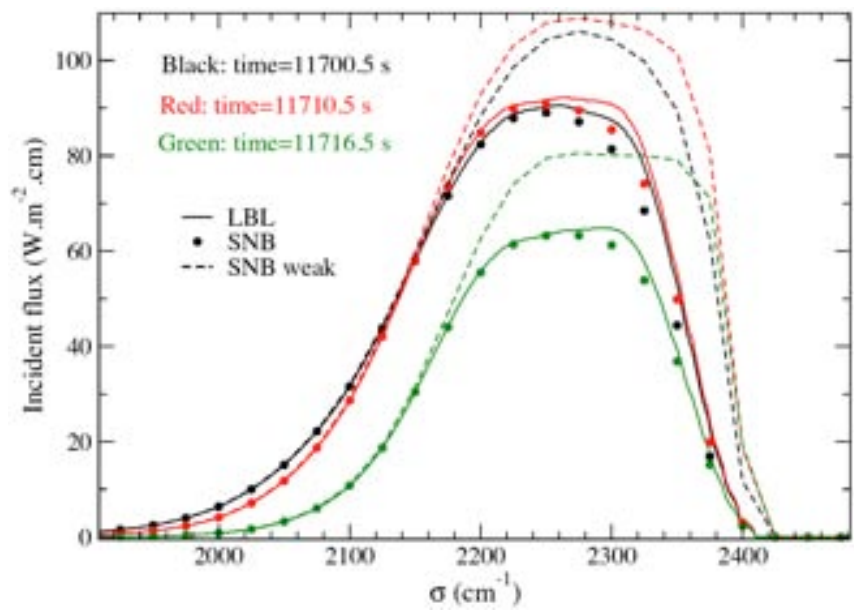

Fig. (17). Low spectral resolution incident flux on a wall point of the outer aluminum cone of the Viking afterbody, calculated using line by line (LBL), SNB model (SNB), and SNB in the weak absorption limit (SNB weak). The three colors refer to three trajectory times. Adapted from [33].

\section{RADIATIVE PROPERTIES FOR JUPITER ATMOSPHERE ENTRY PLASMA}

In this section the model used for the calculation of the spectral radiative properties of the Jupiter atmosphere under high speed shock conditions is described. An important property of the proposed model is that it can be easily coupled to collisional-radiative models (CRM) for the calculation of non-equilibrium spectral coefficients using the actual population of atomic and molecular levels, to determine the absorption coefficient and emissivity entering the radiative transfer equation (RTE). Solving the RTE allows for a self-consistent determination of both the radiative rate coefficients entering the master equations of the CRM and the contribution of radiation to the internal energy of the flow, which could be important in the case of low-pressure, high-speed entry trajectories in atmospheric entry problems [35].

\subsection{Spectral Properties of Jupiter Plasmas}

The present spectral model for the $\mathrm{H}_{2} / \mathrm{He}$ plasma is based on a previous model for pure hydrogen plasma, extended to include the helium atom and molecular hydrogen. The following radiative processes are included:

- Absorption and emission due to transitions between atomic or molecular bound levels (bound-bound transitions) of $\mathrm{H}$, $\mathrm{He}$ and $\mathrm{H}_{2}$ species.

- Transition of the optical electron from a bound state towards the continuum (photoionization) and the reverse process of capture of a free electron by the corresponding ion (radiative recombination, boundfree transitions) of $\mathrm{H}$ and $\mathrm{He}$.

- Bremsstrahlung emission from free electrons in the electric field of the $\mathrm{H}^{+}$ions and inverse Bremsstrahlung absorption (free-free transitions).

The reader is referred to [36] for a full account of the model limited to atomic hydrogen species. Table 1 reports the radiative processes considered in the full model. In the next sections, the implementation of the new radiative processes $R_{2}, R_{3}$ and $R_{5}$ is described.

\subsubsection{Bound-Bound Transitions of $\mathrm{H}_{2}$}

Modeling of $\mathrm{H}_{2}$ Lyman $\left(\mathrm{H}_{2}\left(B^{1} \Sigma_{u}^{+} \leftrightarrow X^{1} \Sigma_{g}^{+}\right)\right)$and Werner $\left(\mathrm{H}_{2}\left(C^{1} \Pi_{u} \leftrightarrow X^{1} \Sigma_{g}^{+}\right)\right)$radiation is performed at the level of rational lines (including $\lambda$-doubling for the $C$ state) using Einstein coefficients $A_{e^{\prime \prime}, v^{\prime \prime}, J^{\prime \prime}}^{e^{\prime}, J^{\prime}}$ and corresponding transition energies calculated by Abgrall et al. in a series of papers, see [42] and references there in. Abgrall's et al. calculations fully account for the non-adiabatic couplings between $\mathrm{H}_{2}$ electronically excited states up to $n=3$, and have been validated by comparison with high-resolution spectroscopic measurements [43]. These data are available online as a part of Paris-Meudon Observatory MOLAT database [38]. This data set is recommended for the calculation of highresolution bound-bound spectra of $\mathrm{H}_{2}$.

Table 1. Radiative processes considered in the calculation of the spectral radiative properties of $\mathrm{H}_{2} / \mathrm{He}$ plasma.

\begin{tabular}{|c|l|c|}
\hline & \multicolumn{1}{|c|}{ Reaction } & Ref. \\
\hline \hline$R_{1}$ & $\mathrm{H}(\mathrm{n}) \rightleftharpoons \mathrm{H}(\mathrm{m}<\mathrm{n})+\rightsquigarrow$ & {$[37]$} \\
\hline$R_{2}$ & $\mathrm{H}_{2}\left(\mathrm{~B}^{1} \Sigma_{\mathrm{u}}^{+}, \mathrm{C}^{1} \Pi_{\mathrm{u}}\right) \rightleftharpoons \mathrm{H}_{2}\left(\mathrm{X}^{1} \Sigma_{\mathrm{g}}^{+}\right)+\rightsquigarrow$ & {$[38]$} \\
\hline$R_{3}$ & $\mathrm{He}(\mathrm{n}) \rightleftharpoons \mathrm{He}(\mathrm{m}<\mathrm{n})+\rightsquigarrow$ & {$[17]$} \\
\hline$R_{4}$ & $\mathrm{H}^{+}+\mathrm{e}^{-}(\varepsilon) \rightleftharpoons \mathrm{H}(\mathrm{n})+\rightsquigarrow$ & {$[39]$} \\
\hline$R_{5}$ & $\mathrm{He}^{+}+\mathrm{e}^{-}(\varepsilon) \rightleftharpoons \mathrm{He}(\mathrm{n})+\rightsquigarrow$ & {$[40]$} \\
\hline$R_{6}$ & $\mathrm{H}^{+}+\mathrm{e}^{-}(\varepsilon) \rightleftharpoons \mathrm{H}^{+}+\mathrm{e}^{-}\left(\varepsilon^{\prime}<\varepsilon\right)+\rightsquigarrow$ & {$[41]$} \\
\hline
\end{tabular}

The calculation of equilibrium spectra, and of nonequilibrium spectra using vibrational populations calculated by a CRM needs accurate partition functions to distribute the total $\mathrm{H}_{2}$ number density (equilibrium) or the vibrational population of the ground and electronically excited states $\mathrm{H}_{2}(Y, v)(\mathrm{CRM})$ over the rotational manifold. The partition function of $\mathrm{H}_{2}$ has been calculated using spectroscopic Dunham coefficients from [44] for the $X$ state and from [45] for the $B$ and $C$ states. It is worth noting that the population of a rotational $J$-level of an homonuclear diatomic molecule depends also on the symmetry of the electronic wave function due to nuclear statistics considerations $[44,46]$. 
Individual rotational line profiles $\left(e^{\prime}, v^{\prime}, J^{\prime}\right)-\left(e^{\prime \prime}, v^{\prime \prime}, J^{\prime \prime}\right)$ have been modeled with an approximate Voigt line shape function [47], using the following expression to determine the Doppler and collisional half-widths [48]:

$$
\begin{aligned}
& \gamma_{D}=\frac{\bar{v}_{0}}{c} \sqrt{\frac{2 \ln 2 k T_{g}}{M}} \\
& \gamma_{\text {coll }}=0.1\left(\frac{273}{T_{g}}\right)^{0.7} \mathrm{~cm}^{-1}
\end{aligned}
$$

\subsubsection{Bound-Bound Transitions of He}

The recent comprensive tabulation by Wiese and Fuhr [49] includes transition probability data for about 2400 transitions with principal quantum number $n \leq 10$ and orbital argular momentum quantum number $\ell \leq 7$, taking fully into account the fine structure. The data set also includes transition probabilities for several intercombinations lines, and is available in NIST's atomic spectral database [17].

The few lines missing from NIST's tables, namely the lines involving the excited states with $n=9, \ell=8$ and $n=10, \ell=8,9$ have been estimated as follows. The dipolar transition probability is given by

$A\left(J^{\prime}, J\right)=\frac{64 \pi^{4}\left(e a_{0}\right)^{2}}{3 h\left(4 \pi \varepsilon_{0}\right)} \bar{v}^{3} \frac{S\left(J^{\prime}, J\right)}{2 J^{\prime}+1}$

The transition strength $S\left(J^{\prime}, J\right)$ (a.u.) can be written as [50]

$S\left(J^{\prime}, J\right)=R_{\text {line }}^{2} R_{\text {multiplet }}^{2} I^{2}$

where the line and multiplet factors are defined as

$$
\begin{aligned}
& R_{\text {line }}=(-1)^{S+J+L^{\prime}+1} \\
& \times \sqrt{(2 J+1)\left(2 J^{\prime}+1\right)}\left\{\begin{array}{ccc}
S & J & L \\
1 & L^{\prime} & J^{\prime}
\end{array}\right\} \\
& R_{\text {multiplet }}=G \times(-1)^{L_{c}+L+\ell^{\prime}+1} \\
& \times \sqrt{(2 L+1)\left(2 L^{\prime}+1\right)}\left\{\begin{array}{ccc}
L_{c} & L & \ell \\
1 & \ell^{\prime} & L^{\prime}
\end{array}\right\}
\end{aligned}
$$

In the latter equation the $L, S, J$ are the spin, orbital and total angular momentum quantum numbers, $L_{c}$ is the orbital angular momentum quantum number of the core, the primed quantities refer to the upper state of the transition, $I$ is the orbital quantum number of the "optical" electron and $G$ is a coefficient (fractional parentage coefficient) that assumes value $G=1$ for transitions of the kind $I \ell-I \ell^{\prime}$ and $G=\sqrt{2}$ for transitions $\ell^{2}-\ell \ell^{\prime}$, where $I$ is a ionic core. In the case of atomic helium, only transitions of the kind $1 s^{2}-1 s . n p$ and 1 s.n $\ell-1$ s.n $\ell \pm 1$ have been considered for calculation with the approximate method. Finally, the radial factor is defined as [51]
$\mathrm{I}=(-1)^{\ell-\ell} \sqrt{\ell}>\int_{0}^{\infty} P_{v \ell}(r) r P_{v^{\prime} \ell^{\prime}}(r) d r$

where $P_{v \ell}(r)$ is a radial function and $\ell_{>}=\max \left(\ell, \ell^{\prime}\right)$. In the Coulomb approximation, the radial functions are replaced by the asymptotically accurate expression

$$
P_{v \ell}(r) \sim K W_{v, l+\frac{1}{2}}(r)
$$

where $K$ is an approximate normalization factor and $W$ is the Wittaker function. In the above equations, $v$ is a generally non-integral effective quantum number, calculated from the experimental level energy $E_{i}$ as

$v=\zeta \sqrt{\frac{\mathrm{Ryd}}{I_{\text {ion }}-E_{i}}}$

where $\zeta=1,2, \ldots$ for neutral atoms, first ions etc., Ryd is the Rydberg constant and $I_{i o n}$ is the experimental ionization energy of the Rydberg series to which the given excited level belongs. The radial integrals have been evaluated according to the method described in section III of [52]. This paper improves over the previous work [36] implementing a method for the calculation of quadratic Stark widths of neutral He lines following [53]. The electron impact Stark half-width $w_{e}$ and shift $d_{e}$ in $\mathrm{cm}^{-1}$ are calculated using:

$$
\begin{aligned}
w_{e}+i d_{e} & =\frac{2}{3 c} n_{e}\left(\frac{\hbar}{m}\right)^{2} \int \frac{d v}{v} f_{e}(v)\left\{\frac{3}{4}\left(\frac{m v}{\hbar}\right)^{2} \rho_{\min }^{2}\right. \\
& +\sum_{i^{\prime}} \mathbf{R}_{i i^{\prime}}^{2}\left[a_{i i^{\prime}}\left(z_{i i^{\prime}}^{\min }\right)-i \varepsilon_{i i^{\prime}} b_{i i^{\prime}}\left(\tilde{z}_{i i^{\prime}}^{\min }\right)\right] \\
& \left.+\sum_{f^{\prime}} \mathbf{R}_{f f^{\prime}}^{2}\left[a_{f f^{\prime}}\left(z_{f f^{\prime}}^{\min }\right)+i \varepsilon_{f f^{\prime}}, b_{f f^{\prime}}\left(\tilde{z}_{f f^{\prime}}^{\min }\right)\right]\right\}
\end{aligned}
$$

where $n_{e}$ is the electron density $f_{e}(v)$ is the eedf, $\rho_{\min }$ is a threshold impact parameter and $R_{j j^{\prime}}$ are coordinate matrix elements that have been calculated from available Einstein coefficients, while the summations on $i^{\prime}$ and $f^{\prime}$ represent the contribution to the broadening of the dipole interaction of the lower and upper states of the line with nearby energy levels and the other quantities are defined as in [53]. The total Stark half-width of a line has been calculated according to [54]:

$w=w_{e}+1.75 A(1-0.75 R) w_{e}$

where $A$ is the ion-broadening parameter calculated according using Eq. (224) of [55] and $R$ is the ratio between the the Debye length and the average ion-ion distance, calculated according to [54].

\subsubsection{Bound-Free Transitions of He}

The most reliable atomic photoionization cross sections (and radiative recombination) calculations up to date are based on the close-coupling (CC) approximation and the Rmatrix method as implemented by the Opacity Project (OP) [24]. Very recently, the cross section data for He in the OP 
database have been superseded by the calculations reported in [56], which are based on the same CC method as the OP database, but using an improved theoretical approach accounting for photoionization and dielectronic recombination in a unified manner. These data include both the total photoionization cross section, leaving the ion $\mathrm{He}^{+}$in various excited core states as well as level-specific photoionization cross sections leaving the ionic core in its ground electronic state. The cross sections are given on a photon energy mesh of a few thousand points, where the lower energy point corresponds to energy difference between the level $i$ and the core ground state. Cross sections are provided for excited states with $n \leq 10$ and $\ell \leq 6$ for both the singlet and triplet systems of He. The cross sections are calculated in the LS coupling scheme and are not resolved over the fine structure, which is not big issue for $\mathrm{He}$ since the fine splittings are anyway very small. This latter dataset has been employed in the present work for the calculation of photoionization/recombination spectra of $\mathrm{He}$ atom.

\subsection{Results}

In this section we present calculated spectra of a Jupiterlike plasma of initial molar composition $\mathrm{H}_{2}: \mathrm{He}=89: 11$. In Fig. (18) absorption spectra of an equilibrium plasma at $p=1 \mathrm{~atm}$ and different temperatures are reported. It is clearly observed that with increasing temperature, molecular absorption lines in the region $60,000-110,000 \mathrm{~cm}^{-1}$ are progressively overcome by atomic $\mathrm{H}$ Lyman absorption. At very high temperature, absorption from atomic $\mathrm{H}$ and $\mathrm{He}$ excited states and Bremsstrahlung below $50,000 \mathrm{~cm}^{-1}$ starts to be significant compared to absorption from the ground states of atoms.

In Fig. (19) absorption spectra calculated at different points in the post-shock region of an hypersonic shock wave propagating in a Jovian plasma $\left(\mathrm{H}_{2}: \mathrm{He}=89: 11\right)$ initially at $T_{0}=160 \mathrm{~K}$ and $P_{0}=10 \mathrm{~Pa}$ and a free stream gas flow speed $v_{0} \approx 46 \mathrm{Km} / \mathrm{s}$. Downstream the shock the gas temperatures suddenly jumps to $T_{g}=81,000 \mathrm{~K}$ and the pressure to $P=2.910^{4} \mathrm{~Pa}(\approx 0.3 \mathrm{~atm})$. The post-shock relaxation of the generated plasma is studied using a CRM [57], which recently has been extended with a reasonably complete set of elementary processes for the $\mathrm{He}$ atom [58]. In Fig. (19), absorption spectra corresponding to the two limiting cases of optically thin (the emitted radiation escapes the plasma volume without being reabsorbed) and optically thick (the emitted radiation is completely reabsorbed locally) are reported. At small distances from the shock front (Fig. 19a) most of the absorption is due to undissociated $\mathrm{H}_{2}$ molecules and $\mathrm{He}$, while at about $20 \mathrm{~cm}$ from the shock front (Fig. 19b), corresponding to the maximum of the electron molar fraction, absorption features of atomic $\mathrm{H}$ Lyman lines are increased due to formation of hydrogen atoms by dissociation of $\mathrm{H}_{2}$ molecules. The thin plasma is characterized by a slightly smaller dissociation degree of $\mathrm{H}_{2}$ and a lower ionization degree, resulting in stronger absorption in $\mathrm{H}_{2}$ bands and smaller Stark widths of atomic
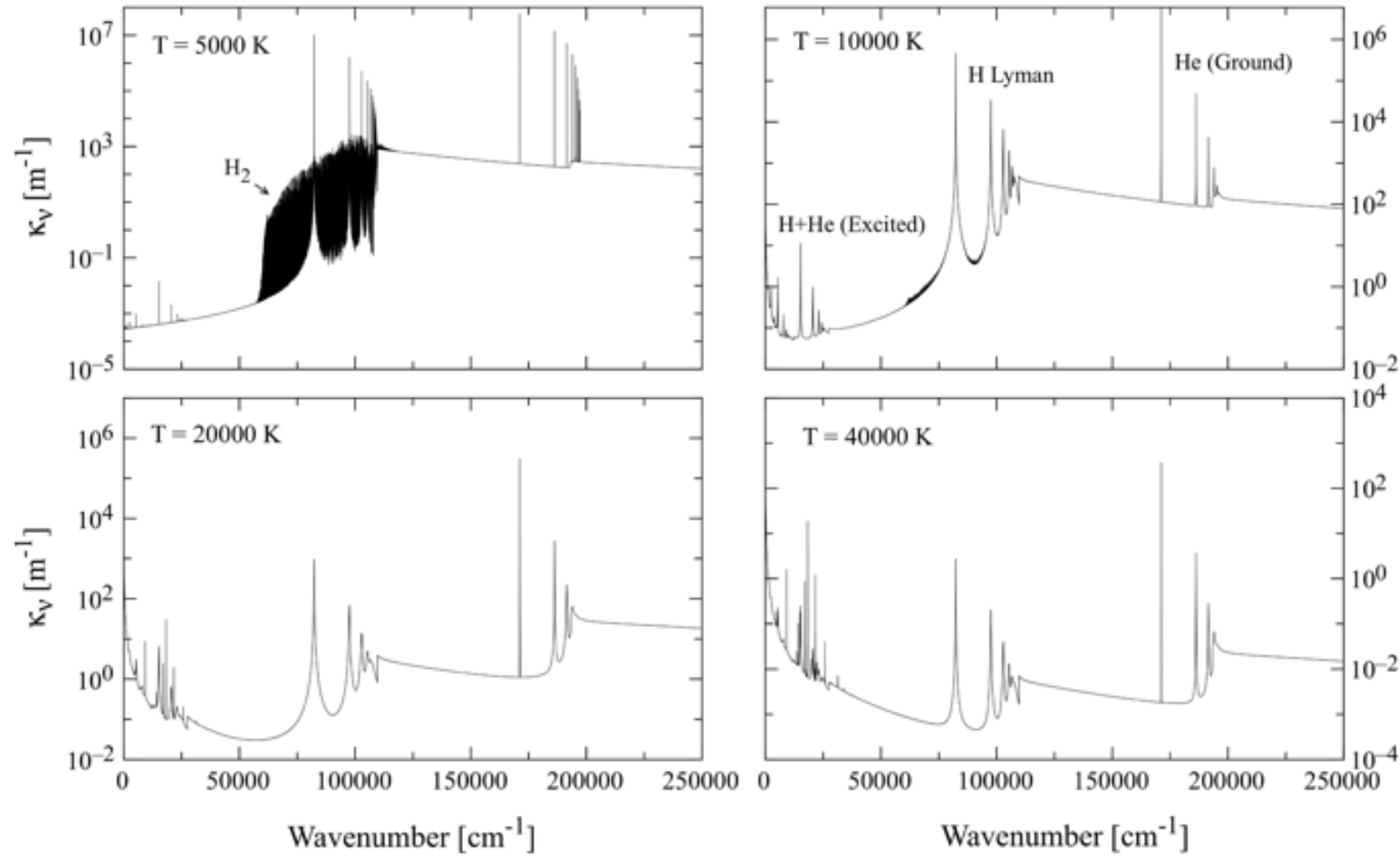

Fig. (18). Calculated absorption spectra of an equilibrium $\mathrm{H}_{2} / \mathrm{He}$ Jovian plasma at $\mathrm{p}=1 \mathrm{~atm}$ at different temperatures. 


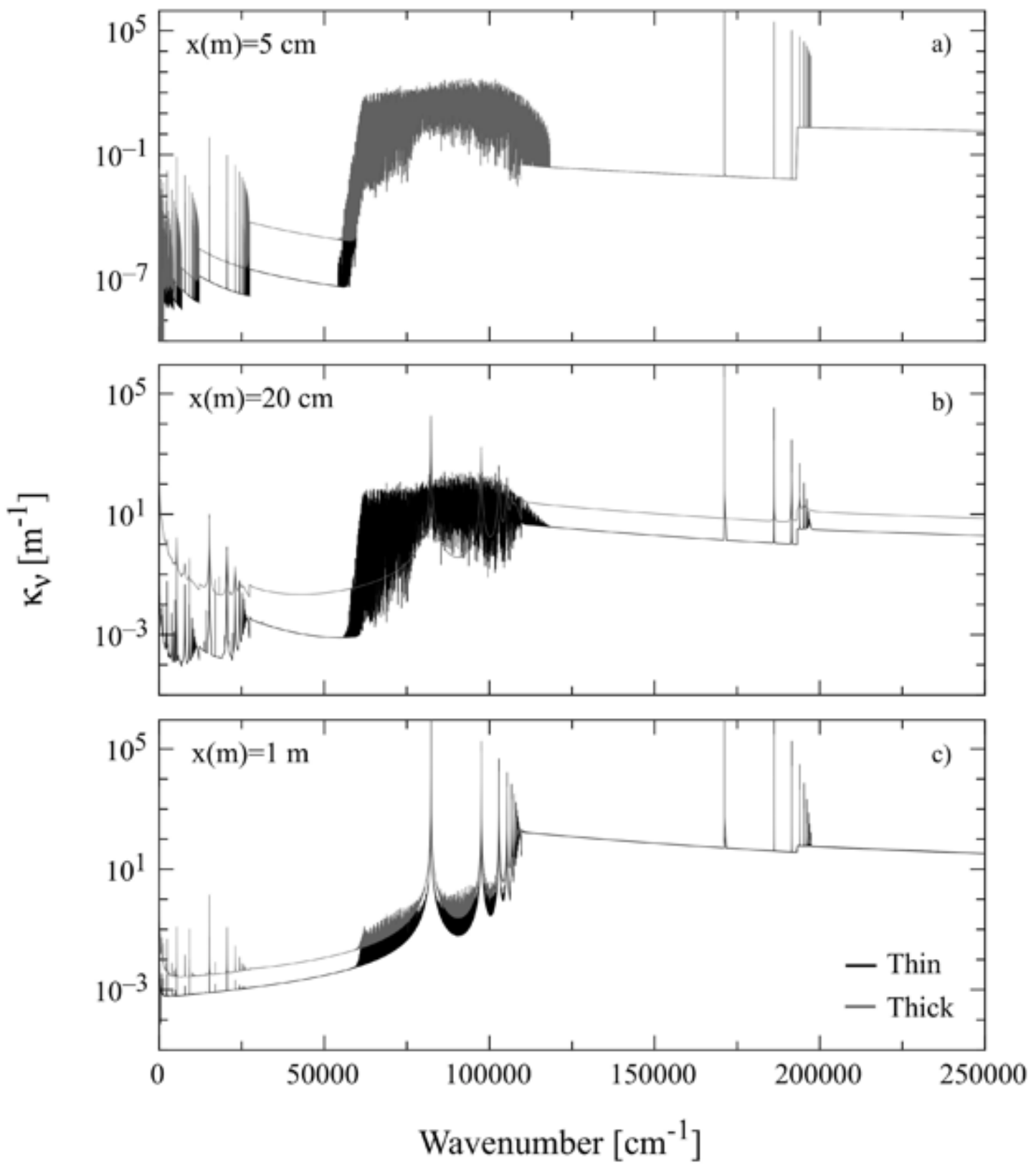

Fig. (19). Calculated absorption spectra of a Jovian plasma at different positions in the post shock region of a shock wave. The initial free stream condition is defined by $T_{0}=160 \mathrm{~K}, P_{0}=10 \mathrm{~Pa}$ and free flow speed $v_{0} \approx 46 \mathrm{Km} / \mathrm{s}$, corresponding to a Mach number $M \approx 50$. Optically thin (solid black line) and optically thick (solid gray line) are compared.

lines. At large distances from the shock front (Fig. 19c) the thin and thick cases are not very different, being both the plasma composition and internal distributions nearly equilibrium at this distance, but the thick case is characterized by stronger absorption below $50,000 \mathrm{~cm}^{-1}$ due to the higher number densities of excited states resulting from neglecting radiative the decay process.

\section{CONCLUSION}

Many advances and improvements have been recently achieved in the field of radiation properties of low temperature plasmas and hypersonic flows. These achievements have been illustrated in this paper with regard to radiation in atmospheric entries, including Earth, Mars, and Jupiter like atmospheres.

A description of the computing code ASTEROID has been presented. This code is applicable to many different kinds of spectral and group optical models for hot gases and low-temperature plasmas. The half-spherical emissivity which is of practical interest for estimation of the radiative heating of space vehicles surface, has been shown to involve a large number of different elementary radiative processes for air plasma.

Using the HTGR database, it has been shown that for entry in Mars atmosphere, two types of radiative processes are predominant. Up to $4000 \mathrm{~K}$, the contribution of $\mathrm{CO}_{2} \mathrm{IR}$ radiation is important. Above and up to $7,000 \mathrm{~K}$, the 
radiation due to diatomic bound-bound transitions dominates. For these two types of processes, an experimental validation has been performed. An example of spectral distribution of the radiative flux on an afterbody has been presented based on line-by-line calculations and on statistical narrow band model whose parameters have been deduced from line-by-line calculations.

Finally, recent improvements of a model for the calculation of radiative properties of high-temperature $\mathrm{H}_{2} / \mathrm{He}$ mixtures, taking into account the most important radiative processes have been presented. It can be used to determine emissivity and absorption coefficient needed in the solution of the radiative transfer equation for hypersonic entry flows, and can be easily coupled to a collisional-radiative model to compute rate coefficients of radiative processes selfconsistently with the radiation field, which can be important in the state-to-state chemical kinetic description of nonequilibrium atmospheric entry plasmas.

To increase the reliability of radiative heating prediction, there is a need for further verification and validation of the presented radiative data. Some additional elementary radiation processes require further investigation. This is the case for instance of processes involving ablation products (when ablative materials are used) which may contain many exotic molecules that can contribute to radiation shielding. Future work should also include the modeling of resonance broadening of He atoms, which is important to predict line widths in low-temperature He-rich plasmas, and the modeling of photodissociation and photoionization of $\mathrm{H}_{2}$ molecules, which could be important to model the chemistry of the precursor region just before a shock front.

The prediction of radiative transfer in entry problems and in hypersonic flows is closely related to the knowledge of the thermodynamic state of the gas surrounding the vehicle. Level populations of the various species must be known with accuracy compatible with the searched accuracy for radiative transfer. These level populations may also be influenced by radiation. Therefore, a consistent treatment of coupled radiation and collision processes is sometimes necessary, though not easy to implement. As an example, the full model on $\mathrm{H}_{2} / \mathrm{He}$ is currently being incorporated in an advanced collisional-radiative model, including a Boltzmann solver for the non-equilibrium electron energy distribution function [59].

\section{CONFLICT OF INTEREST}

The authors confirm that this article content has no conflict of interest.

\section{ACKNOWLEDGEMENTS}

The research leading to these results has received funding from the European Community's Seventh Framework Programme [FP7/2007/2013] under grant agreement no. 242311

\section{REFERENCES}

[1] Whiting EE, Park C, Liu Y, Arnold JO, Paterson JA. NEQAIR96, Non-equilibrium and Equilibrium Radiative Transport and Spectra Program: User's Manual. Reference Publication 1389, NASA, Ames Research Center, Moffet Field, California, Dec 1996

[2] Hartung LC. Predicting radiative heat transfer in thermo-chemical non-equilibrium flowfields: theory and user's manual for the
LORAN code. Technical Memorandum 4564, NASA Langley Research Center, Hampton 1994; VA 23681-0001.

[3] Fujita K, Abe T. SPRADIAN, structural package for radiation analysis: theory and application. Technical report, ISAS Report No 669, 1997.

[4] Kuznetsova LA, Surzhikov ST. Absorption cross sections of diatomic molecules for problems of radiative heat transfer in lowtemperature plasma. High Temp 1999; 37(3): 374-85.

[5] Laux CO. Radiation and non-equilibrium collisinal-radiative models. In VKI special course on physico-chemical models for high enthalpy and plasma flows modeling 2002: June 4-7.

[6] Smith A, Wood A, Dubois J, Fertig M, Pfeiffer B. Plasma radiation database parade v22 final report. Technical Paper 3, ESTEC contract 11148/ 94/NL/FG, FGE TR28/96, 2006.

[7] Johnston C, Hollis B, Sutton K. Non-Boltzmann modeling for air shock-layer radiation at lunar-return conditions. J Spacecr Rockets 2008; 45: 865-78.

[8] Passarinho P, Lino da Silva M. GPRD, a database for the spectral properties of diatomic molecules of atmospheric interest. J Mol Spectrosc 2006; 236(1): 148-9. Available from: http://www. sciencedirect.com/science/article/B6WK8-4J625WX-1/2/8ed0df3b7 1e3410a8accb6372088d6c4

[9] Armstrong BH, Nicholls RW. Emission, absorption and transfer of radiation in heated atmospheres. Oxford: Pergamon Press 1972.

[10] Bates DR, Damgaard A. The calculation of absolute strengths of spectral lines. Philos Trans R Soc 1949; 242: 101-11.

[11] Hartree DR. The calculation of atomic structures. New York: John Wiley Sons, Inc 1957.

[12] Levinson IB, Nikitin AA. Handbook for theoretical computation of line intensities in atomic spectra. New York: Daniel Davey Co., Inc 1965.

[13] Park C. Non-equilibrium hypersonic aerothermo-dynamics. WilleyInterscience Publication, New York 1990.

[14] Surzhikov ST. Computational radiation models for lowtemperature plasma. AIAA 1996; 96-2313.

[15] Surzhikov ST. Computing System for Mathematical Simulation of Selective Radiation Transfer. AIAA 2000; 10.2514/6.2000-2369.

[16] Moore CE. Atomic energy levels. NBS Circular 467, Washington D.C, Vol. 1, 1949; Vol. 2: 1952; Vol. 3, 1958

[17] Kramida A, Ralchenko Yu, Reader J, and NIST ASD Team. NIST Atomic Spectra Database (version 5.0). National Institute of Standards and Technology: Gaithersburg, MD 2012.

[18] Huber KP, Herzberg G. Molecular Spectra and Molecular Structure. IV. Constants of Diatomic Molecules. New York: VanNostrand-Reinhold Company 1979.

[19] Hayes WD, Probstein RF. Hypersonic flow theory. hypersonic flow theory. New York and London 1959.

[20] Chauveau S, Perrin MY, Rivière P, Soufiani A. Contributions of diatomic molecular electronic systems to heated air radiation. J Quant Spectrosc Radiat Transf 2002; 72: 503-30.

[21] Chauveau S, Deron C, Perrin MY, Rivière P, Soufiani A. Radiative transfer in LTE air plasmas for temperatures up to $15,000 \mathrm{~K}$. J Quant Spectrosc Radiat Transf 2003; 77:113-30.

[22] Babou Y, Rivière P, Perrin MY, Soufiani A. Spectroscopic data for the prediction of radiative transfer in $\mathrm{CO}_{2}-\mathrm{N}_{2}$ plasmas. J Quant Spectrosc Radiat Transf 2009; 110: 89-108.

[23] Perrin MY, Rivière P, Soufiani A. Radiation phenomena behind shock waves. In: Brun R, Ed. High temperature phenomena in shock waves. Springer-Verlag: USA 2012: pp. 193-230.

[24] OP Team. The Iron Project - The Opacity Project 2012. Available from: http: //cdsweb.u-strasbg.fr/topbase/topbase.html

[25] Tashkun SA, Perevalov VI, Coudert LH. Cdsd-4000: Highresolution, high-temperature carbon dioxide spectroscopic databank. J Quant Spectrosc Radiat Transf 2011; 112(9): 1403-10.

[26] Babou Y, Rivière P, Perrin MY, Soufiani A. Spectroscopic study of microwave plasmas of $\mathrm{CO}_{2}$ and $\mathrm{CO}_{2}-\mathrm{N}_{2}$ mixtures at atmospheric pressure. Plasma Sources Sci Technol 2008; 17 (4): 045010. Available from: $\mathrm{http}: / /$ stacks.iop.org/0963-0252/17/i=4/a= 045010

[27] Depraz S, Perrin MY, Soufiani A. Infrared emission spectroscopy of $\mathrm{CO}_{2}$ at high temperature. Part I: Experimental setup and source characterization. J Quant Spectrosc Radiat Transf 2012; 113: 1-13.

[28] Depraz S, Perrin MY, Rivière P, Soufiani A. Infrared emission spectroscopy of $\mathrm{CO}_{2}$ at high temperature. Part II: Experimental results and comparisons with spectroscopic databases. J Quant Spectrosc Radiat Transf 2012; 113: 14-25. 
[29] Scutaru D, Rosenmann L, Taine J. Approximate intensities of hot $\mathrm{CO}_{2}$ hot bands at 2.7, 4.3 and $12 \mu \mathrm{m}$ for high temperature and medium applications. J Quant Spectrosc Radiat Transf 1994; 52: 765-81.

[30] Goody RM, Yung YL. Atmospheric radiation. Oxford University Press: UK 1989; p. 2.

[31] Rivière $\mathrm{P}$, Soufiani A. Updated band model parameters for $\mathrm{H}_{2} \mathrm{O}$, $\mathrm{CO}_{2}, \mathrm{CH}_{4}$ and $\mathrm{CO}$ radiation at high temperature. Int $\mathrm{J}$ Heat Mass Transf 2012 55: 3349-58.

[32] Rivière P, Perrin MY, Soufiani A. New developments for $\mathrm{CO}_{2} \mathrm{IR}$ radiation: comparison between experiments and spectroscopic database predictions, and updated band model parameters. In: International Workshop on Radiation of High Temperature Gases in Atmospheric Entry, Barcelona 2012, ESA SP-714, December 2012.

[33] Rivière P, Soufiani A. Contribution to test case TC7: line-by-line and SNB calculations of the incident radiative flux on two points on the afterbody. In: Proceedings of the International Workshop on Radiation of High Temperature Gases in Atmospheric Entry, Barcelona 2012; ESA SP-714.

[34] Beck J, Merrifield J. Viking radiation heat flux computation, Test Case 7. In: Proceedings of the International Workshop on Radiation of High Temperature Gases in Atmospheric Entry, Barcelona 2012; ESA SP-714.

[35] Colonna G, Pietanza LD, D'Ammando G. Self-consistent collisional-radiative model for hydrogen atoms: Atom-atom interaction and radiation transport. Chem Phys 2012; 398: 37-45.

[36] D'Ammando G, Pietanza LD, Colonna G, Longo S, Capitelli M. Modelling spectral properties of non-equilibrium atomic hydrogen plasma. Spectrochim Acta B 2010; 65(2): 120-9.

[37] Bautista-Moedano M, Lopez-Bonilla J. Matrix elements $\left(a_{d} \ell_{2}\left|z^{6}\right| u_{1}\right)$ for the Coulomb interaction. Apeiron 2006; 13(1): 34-42.

[38] Paris-Meudon Observatory. MOLAT-Atomic and Molecular Data 2012. Available from:

http://molat.obspm.fr/index.php?page=accueil.php

[39] Storey PJ, Hummer DG. Fast computer evaluation of radiative properties of hydrogenic systems. Comp Phys Commun 1991; 66(1): 129-41.

[40] Nahar SN. NORAD-Atomic-Data (Nahar-OSU-Radiative-AtomicData) 2012. Available from: URL http://www.astronomy. ohiostate.edu/ nahar/nahar radiativeatomicdata/index.html

[41] Karzas WJ, Latter R. Electron radiative transitions in a coulomb field. Astrophys J Suppl S 1961; 6: 167-212.

[42] Abgrall H, Roueff E, Launay F, Roncin JY, Subtil JL. The lyman and werner band systems of molecular hydrogen. J Mol Spectrosc 1993; 157: 512-23.

[43] Liu X, Ahmed SM, Multari RA, Geoffrey KJ, Ajello JM. High resoluton electron-impact study of the far-ultraviolet emission spectrum of molecular hydrogen. Astrophys J Suppl S 1995; 101: 375-99.
[44] Irwin AW. Refined diatomic partition functions. I - Calculational methods and $\mathrm{H}_{2}$ and $\mathrm{CO}$ results. Astron Astropys 1987; 182(2): 348-58.

[45] Dabrowski I. The Lyman and Werner bands of $\mathrm{H}_{2}$. Can J Phys 1984; 62: 1639-64.

[46] Capitelli M, Colonna G, D'Angola A. Fundamental Aspects of Plasma Chemical Physics: Thermodynamics. New York: Springer 2012

[47] Wells RJ. Rapid approximation to the Voigt/Faddeeva function and its derivatives. J Quant Spectrosc Ra 1999; 62(1): 29-48.

[48] Perrin MY, Riviere P, Soufiani A. Radiation Database for Earth and Mars Entry. In: RTO-EN-AVT-162. NATO Science and Technology Organization 2009.

[49] Wiese WL, Fuhr JR. Accurate atomic transition probabilities for hydrogen, helium and litium. J Phys Chem Ref Data 2009; 38(3): 565-719.

[50] Shore WB, Menzel DH. Generalized tables for the calculation of Dipole transition probabilities. Astrophys J Suppl S 1965; 12:187213.

[51] Oertel GK, Shomo LP. Tables for the calculation of radial multipole matrix elements by the coulomb approximation. Astrophys J Suppl S 1968; 16: 175-218.

[52] Klarsfeld S. Alternative forms of the Coulomb approximation for bound-bound multipole transitions. Phys Rev A 1989; 39(5): 2324 32.

[53] Dimitrijevic MS, Konjevic N. Simple formulae for estimating stark widths and shifts of neutral atom lines. Astron Astrophys 1986; 163: 297-300.

[54] Menart J, Heberlein J, Pfender E. Line-by-line method of calculating emission coefficients for thermal plasmas consisting of monoatomic species. J Quant Spectrosc Radiat Transf 1996; 56(3): 377-98.

[55] Griem HR. Spectral line broadening by plasmas. Academic Press New York 1974

[56] Nahar SN. Photoionization and electron-ion recombination of He I. New Astronomy 2010; 15: 417-26.

[57] Colonna G, D'Ammando G, Pietanza LD, Capitelli M. Selfconsistent coupling of chemical, electron and radiation models for shock wave in jupiter atmosphere. In: AIP Conference Proceedings 2012; 1501: 1400-7.

[58] Capitelli M, Colonna G, Pietanza LD, D'Ammando G. Coupling of radiation, excited states and electron energy distribution function in non-equilibrium hydrogen plasmas. Spectrochim Acta B 2013; (in press).

[59] Pietanza LD, Colonna G, De Giacomo A, Capitelli M. Kinetic processes for laser induced plasma diagnostic: A collisionalradiative model approach. Spectrochim Acta B 2010; 65(8): 61626. 